\title{
Surrogacy; A Regression to A PATriarChal SOCIETY FREE OF SOCIAL POLICIES?
}

\author{
LA MATERNIDAD SUBROGADA; ¿UNA REGRESIÓN \\ A UNA SOCIEDAD PATRIARCAL EXENTA DE \\ POLÍTICAS SOCIALES?
}

\begin{abstract}
Contrary to what can be believed, and despite the new techniques of human reproduction, gestational surrogacy is not an exclusive practice of the 21 st century and Roman law already provides us with a series of sources on the use of this practice among the Roman aristocracy. In this work some of these examples are brought up, showing the reason why this practice was carried out in a patriarchal society, which used women for reproductive purposes in the interest not only of the family but also of society itself. This makes us wonder if, nowadays, the use of this practice also responds to social interests, in which women are the object of commodification, perhaps more characteristic of a patriarchal society.

\section{RESUMEN}

En contra de lo que se pueda creer, y a pesar de las nuevas técnicas de reproducción humana, la gestación por sustitución no es una práctica exclusiva del siglo XXI y ya el Derecho Romano nos suministra una serie de fuentes sobre el uso de esa práctica entre la aristocracia romana. En este trabajo se traen a colación algunos de esos ejemplos, mostrando además cual era la razón de que se realizara esta práctica, en una sociedad patriarcal, que utilizaba a la mujer con fines reproductivos en interés no sólo de la familia, sino de la propia sociedad. Esto hace que nos preguntemos si, hoy dia, el uso de esta práctica también responde a unos intereses sociales, en los que la mujer, es objeto de mercantilización.
\end{abstract}

KEYWORDS: Roman law, Surrogacy, patriarchal society, commoditization, precedents 
Palabras Clave: Gestación por sustitución, Derecho Romano, precedentes, sociedad patriarcal, mercantiizacion

La serie televisiva, El cuento de la criada (The handmaid's tale), basada en la novela que Margaret Atwood publicó en 1984 ha sido una de las series con más éxito de este año, y 33 años después de la publicación del libro, se ha puesto de manifiesto, una vez más, que los temas que abordan la crítica social y el tratamiento de la mujer, no están ni mucho menos superados.

La serie, al igual que la novela, refleja una sociedad futura imaginaria en la que un nuevo gobierno totalitario, teocrático y militar tiene como objetivo principal evitar la erradicación de la raza humana, que se ha visto comprometida a través de la creciente infertilidad de las mujeres, derivada en parte del incremento de la contaminación ambiental. Para ello, las mujeres son sometidas y catalogadas como ganado: las infértiles son enviadas a colonias - una especie de campos de concentración- a limpiar y las pocas mujeres fértiles que quedan, llamadas "handmaids" (criadas) de acuerdo a una "interpretación extremista (Cambra Badi, 2018, p. 1) de un versículo de la Biblia, Génesis 30,1-3, sobre el que luego volveremos, son entrenadas, y educadas para 'servir' a los señores y sus esposas como vientres de alquiler.

La utilización del cuerpo de la mujer entronca directamente con el debate actual y abierto, de la maternidad subrogada, o gestación por sustitución, -la elección del término, como apunta Allis (1997, pp. 21-22), no es baladí1. Se apela al derecho de decidir libremente, pero se olvida explicar cómo una mujer va a elegir libremente entre pobreza o ser un vientre de alquiler. La serie evidencia de manera extraordinaria la interseccionalidad entre clase y género. Como las mujeres pobres son las más afectadas, las que apenas tienen derecho. Solo las fértiles pueden salir adelante puesto que sirven de mercancía para las mujeres y hombres heterosexuales ricos, que no pueden tener hijos.

Vemos pues que el Cuento de la criada se hace eco, de forma explícita de la característica distintiva del sistema patriarcal respecto de la concepción de la mujer: el mandato social de la maternidad (Cambra Badi, 2018, pp. 3-4), sistemas estos, en los que la reproducción se considera como una función social básica, donde las mujeres juegan un papel indispensable. 
En el momento del estreno de esta serie, hace ahora un año, se celebró en de ley en ese sentido.

Mientras tanto, las revistas informan de los viajes de famosos para recoger a sus niños, como es el caso de Sir Elton John, Sarah-Jessica Parker, Nicole Kidman, Sharon Stone, Michael Jackson, Dennis Quaid, Ricky Martin, o Cristiano Ronaldo.

A pesar de que en esta práctica, puede haber contraprestación económica o realizarse de forma gratuita, la mayoría de las veces es un servicio que se paga, lo que además, no parece asumible en un futuro próximo por ningún sistema de salud pública (Palmero, 2017, p. 39). Esto hace que se trate de un asunto exclusivo para gente adinerada, lo que al margen de las cuestiones jurídicas, produce desigualdades de clase social e incluso de localización geográfica ya que la tendencia es que las parejas de los países desarrollados contraten los servicios, vía agencias mediadoras, de mujeres de los países más pobres.

En países como EE.UU. las mujeres que optan por la gestación subrogada lo hacen, en muchas ocasiones, para lograr los fondos necesarios para pagar la carísima educación universitaria de los hijos que ya tienen o para afrontar las facturas médicas de sus familiares dado el elevado coste de los seguros médicos privados. Por contra, en países menos desarrollados, son los maridos de estas mujeres los que se apropian de los ingresos derivados de esta práctica. Lo que, nos acerca a las sociedades patriarcales en que las que se protegen los intereses de los hombres, relegando a la mujer a una mera función reproductora. El que en la actualidad se discuta, no sólo sobre el rol que tiene la mujer en las familias monoparentales y homoparentales, las nuevas técnicas de reproducción humana asistida, o incluso en la clonación, sino también sobre las modificaciones que todas estas prácticas producen en el seno de la institución familiar, me hace volver la vista atrás, y retomando el título del presente trabajo, preguntarme si con la maternidad subrogada se produce una regresión a una sociedad patriarcal en la que la mujer tiene un papel indispensable en la procreación y es utilizada para tal fin.

Y la sociedad romana, se caracteriza, precisamente, por ser patriarcal y, basada en la figura del paterfamilias como "jefe" indiscutible de la familia, sólo se preocupa de proteger sus intereses: y si el Derecho es fiel reflejo de la sociedad, a finales de la República Romana el matrimonio era considerado 
según Mommsen, como una carga digna de asumirse solamente en interés público; Tanto fue así, que las nefastas repercusiones del ocaso republicano, no tardaron en hacerse sentir en el propio seno familiar, por cuanto el matrimonio era considerado como una carga pública pesada, que todo patriota debía sobrellevar como un deber, mientras que el divorcio, se convirtió en un "acontecimiento natural y frecuente" como consecuencia del individualismo (Bazan, p. 286).

Es comunis opinio entre la romanística que la finalidad de la unión marital no era otra que la procreatio filiorum, si bien en Derecho romano clásico no aparece de modo explícito. Así se desprende de la tristeza de algunas matronas romanas que no pudieron tener hijos, como en el conocido caso de Turia, que conocemos por la famosa laudatio pronunciada por su marido con ocasión de su muerte en el año 4, en plena época augustea: Mujer casta y respetuosa, no consiguió darle un hijo a su marido, y era tal su nobleza que llegó a proponerle que se divorciara de ella, ofreciéndose a abandonar el hogar a la fecundidad de otra mujer y a aceptar a los hijos que tuviera de esta como propios, proposición que el marido rechazó (Laudatio quae dicitur Turiae en Fontes Iuris romani antiqui III (Negotia).

A tal punto era imprescindible dejar descendencia, que la esterilidad de la mujer se constituyó en una causa de divorcio, como en el caso de Spurio Carvilio Ruga (aproximadamente en el año 230 a.C.) narrado por Aulo Gelio, en sus Noches Áticas que repudió a su esposa, estando profundamente enamorado de ella porque no podía tener hijos.

En definitiva, esa necesidad de procrear no es nueva, y si bien es cierto que son las nuevas técnicas de reproducción asistida las que dan lugar a nuevos medios para formar una familia, como el nacimiento del primer bebé probeta en julio de 1978, o la primera gestación subrogada comercial en 1980; esa mirada al pasado nos muestra que esta última se remonta a varios miles de años atrás, cuando en Mesopotamia era frecuente que las mujeres estériles acudieran a la hoy llamada subrogación tradicional para no ser marginadas y apartadas de la sociedad por no ser capaces de engendrar, permitiéndoseles ofrecer una sierva a sus esposos y reconocer como propios a los hijos nacidos de dicha unión. Así, como ya apuntamos al principio de este trabajo, encontramos referencias expresas en el Antiguo Testamento de 
la Biblia, dónde Sara (Génesis 16, 1-4), ante la imposibilidad de darle hijos a su esposo, el profeta Abraham, le dijo a éste: «Mira, Yahveh me ha hecho estéril. Llégate, pues, te ruego, a mi esclava. Quizá podré tener hijos de ella». O Raquel (Génesis 30, 1-6) que, tras el nacimiento del hijo que su esposo Jacob concibió con su criada, manifestó: «Dios me ha hecho justicia, pues ha oído mi voz y me ha dado un hijo». De ahí, recordemos el nombre de criadas de la novela y serie.

El primer caso de lo que hoy llamamos gestación subrogada tiene escasos 40 años, y se celebró en 1976 en Michigan (EEUU), y bajo la supervisión del abogado Noel Keane se firmó el primer contrato formal entre una madre subrogada y una pareja comitente, si bien se trataba de lo que hoy se llama modalidad "tradicional" de gestación subrogada, es decir, aquella en que la mujer gestante aportará tanto su útero, como sus propios óvulos que serán fecundados; y "altruista”, en la que no habrá contraprestación económica. Esta modalidad, como ya he dicho, no es la más frecuente hoy en día, y sin embargo es la que encontramos en las fuentes romanas, a pesar de que en los ejemplos que nos suministran las fuentes, hay lógicamente, una relación carnal entre el hombre y la mujer.

El caso más destacado y conocido es el de Marcia, Catón y Hortensio, caso denominado por algunos autores como el "extraño triángulo" (Cantarella, 1997, p. 141), y recogido por Plutarco, Caton Minor 25, y Lucano, Pharsalia, II, 331; Comenta Lucani, II, 33; y Adnotationes Lucani, II, 339.

Los personajes son efectivamente tres: Catón de Utica (el marido); Marcia (su esposa) y Quinto Hortensio Hortalo (el tercero), aunque como veremos, la hija de Catón -Porcia- también juega un papel destacable en nuestro caso. La Historia se desarrolla en el año 56 a.C., esto es, a finales de la época Republicana, cuestión a tener en cuenta por las concepciones sobre el matrimonio y el divorcio del momento, y por el panorama político y social reinante y al que ya me he referido.

Catón y Hortensio eran buenos amigos. Además Hortensio admiraba a Catón de tal manera que deseoso de ligarse a su familia, con vínculos de sangre, le solicita como mujer a su hija Porcia, entonces casada con Marco Calpurnio Bíbulo, cónsul en el 59 a.C., con el que ya tenía dos hijos.

Para convencer a Catón, Hortensio, esgrimió el siguiente argumento: 
Si Porcia le hubiese dado un heredero, el [Hortensio] estaría más estrechamente unido a Catón y Bíbulo por esta comunidad de hijos. Lo único que quería Hortensio es que ésta le diera un hijo, y una vez alcanzado el objetivo la devolvería a su marido.

Catón rechazó la propuesta de Hortensio, pero este, no dándose por vencido, propuso a aquel que le cediera a Marcia, su propia esposa, lo bastante joven aun para darle hijos tanto más cuanto la sucesión de Catón ya estaba asegurada (ya tenían 2 hijos).

$\mathrm{El}$ argumento esgrimido por Hortensio Hortalo para convencer a Catón esta vez, fue que si a los ojos de los hombres un hecho similar podría parecer extraño, desde un punto de vista de la naturaleza, resultaba bien hecho y útil a la comunidad el que una mujer, en la flor de la edad y de la belleza, no resultara inactiva, y no dejara marchitar su propia fecundidad; pero lo que no es hermoso es que proporcione dificultades al marido dándole más hijos que los necesarios.

Catón, frente a esta nueva petición duda y consulta con su suegro, Lucio Marcio Filipo, padre de Marcia, que acepta. Curiosamente, a Marcia nadie le pregunta. Así, decidiéndose al final, Marcia se convierte en esposa de Hortensio en el año 56 a.C., dándole dos hijos. A la muerte de Hortensio, 6 años después, Catón vuelve a tomar, consigo a Marcia, como dice Apiano, Bell. civ., II, 14.9. como si la hubiera prestado, todavía joven y ahora además viuda rica (CANTARELLA, 1997, p. 146).

Lo primero que nos llama la atención de esta historia, es que en las dos proposiciones de Hortensio, tanto en la de casarse con Porcia, como en la de casarse con Marcia, ambas mujeres estaban ya casadas.

En el caso de Porcia, el que la hija de Catón estuviera casada, parece ser que no era un problema, sino y más importante, una práctica habitual, ya que al menos hasta el siglo II, los padres podían interrumpir el matrimonio de sus hijas o hijos cuando quisieran, como pone de manifiesto Plutarco, precisamente por eso era requisito indispensable para poder contraer matrimonio el consentimiento del paterfamilias si los contrayentes eran alieni iuris.

La singularidad del caso de Marcia, más allá de la forma jurídica en la que se realiza, hoy sorprende por la mentalidad que revela, ya que muestra 
una ideología y unas costumbres familiares diferentes a las nuestras. Sin embargo el tono en que las fuentes lo refieren es absolutamente normal, sin escándalos, sin reprobaciones, sin desconcierto..., a pesar de tratarse de un suceso que fue muy difundido en la antigüedad pero más por la notoriedad de sus protagonistas (recordemos que Catón llego a ser Pretor de Roma, y que Hortensio fue un prestigioso orador), que por lo que hicieron.

Los protagonistas, personas maduras, habiendo superado el romanticismo juvenil tenían una visión moderada del matrimonio acorde a la época, entendiéndolo como una institución que sirve para dar continuidad al Estado: en efecto, para Catón el matrimonio no era más que el instrumento para asegurarse una descendencia (Lucano 2,387 s) y cumplir así su deber con el Estado; desde el momento en que Marcia ya le había dado varios hijos, este, considerando el valor social, además del afectivo privado, aceptó la solicitud del amigo y le cedió la mujer fértil (Plutarco, Caton min. 25.11), divorciándose de ella.

Para entender el matrimonio romano hay que afrontarlo como una alianza entre dos familias concluido por razones económicas, sociales o políticas y por el deber de organizar la reproducción de los grupos familiares. Sólo desde esta óptica se puede entender la decisión de los maridos de ceder a sus mujeres cuando éstas eran ventres: úteros colmados.

Así, vemos que la uxoris cessio, o cesión de la propia mujer era un práctica habitual entre las clases altas, y que era frecuente que si el marido ya había tenido un número suficiente de hijos, cediera a la propia esposa a otro hombre con el único propósito de que le diera hijos; así se estrechaban además las alianzas sociales y políticas, no sólo en relación a intereses personales o familiares, sino en el marco de los intereses de la ciudad, es decir para cumplir con ese deber de Estado del que hemos hablado. Observamos así que los deberes públicos de la matrona romana no se circunscriben a su propia familia, sino que trascienden al interés público.

Plutarco, habla de que el marido romano en caso de tener un número suficiente de hijos, y si otro que deseaba tenerlos lo convencía, se separaba de la mujer y se la cedía, sin perder la manus o la potestad sobre ella. Una vez cumplido el objetivo, el marido podía recuperar a la mujer o no. Y la mujer, como buena ciudadana, aceptaba desarrollar esta función, prueba de ello es 
que Marcia vuelve a contraer matrimonio con Catón, al que parece que no guardaba ningún rencor.

El marco jurídico en el que se realizaba esta cesión de la esposa, podía ser sin divorciarse del marido, y por tanto continuaban casadas con el primer marido, o divorciándose de él, como en el caso que nos ocupa.

Reflexionando a cerca de este supuesto (el de Marcia), observamos la presencia de notas típicas de la gestación por sustitución como lo son el ofrecimiento de un óvulo y la prestación del vientre realizados por la mujer, convirtiéndose en madre biológica y genética del niño (modalidad tradicional), con la salvedad de que en la hipótesis planteada, los progenitores se unieron en legítimo matrimonio previo divorcio de la donante. Por su parte, el requirente asumiría los derechos y deberes derivados de la procreación. En ésta posición Hortensio sería no solo el padre genético, sino también biológico del nacido.

En el de caso de Porcia, hubiera sido lo mismo: si Catón, su padre, hubiera accedido a la pretensión de Hortensio, se hubiese configurado en efecto, lo que hoy se conoce como gestación por sustitución, pues Hortensio se comprometía a restituir a la joven a su esposo Bíbulo después de haber parido. Así, la presunta madre hubiese donado un óvulo y su vientre, convirtiéndose en madre biológica y genética del niño.

Un dato (casi siempre olvidado) que no hemos tenido en cuenta hasta ahora, y que a nuestro juicio es el más interesante de todo esto, es el hecho de que Marcia, cuando fue entregada a Hortensio ya estaba embarazada, con lo que uno de los hijos frutos de ese matrimonio, era de Catón. Parece ser que efectivamente, los romanos no solo solían ceder a sus mujeres, sino también que solían hacerlo en el momento en que ya estaban embarazadas: otros casos de personajes conocidos son el de Sila, que hizo que su hija Emilia (hija de su $4^{\text {a }}$ mujer, Metella con su primer marido, Q. Emilio Escauro), se divorciara de su primer marido para casarse ya embarazada, con Gneo Pompeyo (81aC), o Livia, esposa de Tibero Claudio Nerón, y madre de Tiberio, que embarazada de su segundo hijo Druso, fue cedida a Augusto. (Caso muy polémico ya que parece ser que Augusto la robó).

Todos estos casos, a mi juicio, también pueden encajarse en la hoy llamada gestación por sustitución, a pesar de que el material genético masculino 
tampoco fuera del hombre (marido en nuestro caso), porque como recoge la definición comúnmente aceptada por la doctrina que resulta de la sentencia n. 826 de la Sección 10. ${ }^{\text {a }}$ de la Audiencia Provincial de Valencia, de 23 de noviembre de 2011, la gestación por sustitución «consiste en un contrato, oneroso o gratuito, a través del cual una mujer consiente en llevar a cabo la gestación, mediante técnicas de reproducción asistida, aportando o no su óvulo, con el compromiso de entregar el nacido a los comitentes, que pueden ser una persona o una pareja, casada entre sí o no, que a su vez pueden aportar o no sus gametos».

En síntesis, una vez más vemos que Roma, sin contar con tecnologías sofisticadas, nos suministra una serie de ejemplos y casos que permitían alcanzar los resultados que hoy se alcanzan con la maternidad subrogada. Del mismo modo, El cuento de la criada, al que aludíamos al principio, a pesar de ser un relato distópico y de ciencia ficción, aquello que retrata no está tan alejado de las problemáticas con las que nos enfrentamos en la actualidad.

En definitiva, y al hilo del título de nuestro trabajo; ¿es hoy la maternidad subrogada, como lo fue en Roma, y lo es en la novela, un reflejo de una sociedad patriarcal que considera la reproducción como una función social básica, y en la que la mujer es usada para tal fin?

\section{Referencias}

Allis, T. (1997). The moral implications of motherhood by hire, Medical Ethics Vol. 5 No. 1 Jan-Mar, pp. 21-22.

Bazàn, M.E. y Llaryora, B., Maternidad subrogada -¿existió en Roma?, pp. 285-293.

Cambra Badi, et alii. (2018). Cuestiones bioéticas y biopolíticas en la serie El cuento de la criada. Rev. Med. Cine [Internet];14(x): pp. 1-11.

Cantarella, E. (1997). Pasado Próximo. Mujeres romanas de Tácita a Sulpicia. Madrid: Universitat de València. ISBN 8437615720, 9788437615721.

Guerra Palmero, M.J. (2018). Contra la mercantilización de los cuerpos de las mujeres. La "gestación subrogada" como nuevo negocio transnacional, en Rodríguez Delgado, Janet: Vulnerabilidad, justicia y salud global. Dilemata, Revista Internacional de Éticas Aplicadas, año 10, n. ${ }^{\circ}$ 26, 39-51. ISSN 0034-8252. 


\section{Endnotes}

${ }^{1}$ Somos conscientes de que la utilización de los términos maternidad o gestación por sustitución puede hacer pensar en una notoria ambigüedad. Sin embargo, del mismo modo queremos destacar la carga valorativa que se desprende de cada uno de ellos. En este trabajo, dado que tiene un contenido eminentemente jurídico, utilizamos mayoritariamente el de gestación por sustitución que es el término legal en España tal como recoge el art. 10 de la Ley de Técnicas de Reproducción Humana Asistida de 2006. Sin embargo, dado en marco en el que nos encontramos y en el que se ha desarrollado esta comunicación, y al hilo de nuestras reflexiones finales, hemos querido destacar en el título de nuestro trabajo el de maternidad por contribuir al objetivo de la Conferencia de analizar experiencias y casos de especial interés a partir de las raíces romanas, para así poder contribuir a estimular el debate sobre los procesos de transformación de los escenarios de acción gubernamentales. 